\title{
Overstress and flowstress approaches to dynamic viscoplasticity
}

\author{
Yehuda Partom ${ }^{\mathrm{a}}$ \\ Retired from RAFAEL, Israel
}

\begin{abstract}
Viscoplasticity is mostly modelled by the flowstress approach, where the flowstress (Y) is a function of pressure, temperature, plastic strain and strain rate $\mathrm{Y}\left(\mathrm{P}, \mathrm{T}, \varepsilon^{p}, \dot{\varepsilon}\right)$. For dynamic Viscoplasticity the flowstress approach is used in hydrocodes together with the radial return algorithm, to determine deviatoric stress components in each computational cell and for each time step. The flowstress approach assumes that during plastic loading, the flowstress in stress space follows the current stress point (current Y). Unloading of a computational cell is therefore always elastic. The overstress approach to dynamic viscoplasticity was used in various versions in the 1950s and early 1960s, before the advent of hydrocodes. By the overstress approach a state point may move out of the quasistatic flow surface upon loading, and hence the term overstress. When this happens, the state point tends to fall back (or relax) onto the quasistatic flow surface through plastic flow, and the rate of this relaxation is an increasing function of the amount of overstress. In the paper we first outline in detail how these two approaches to dynamic viscoplasticity work, and then show an example for which the overstress approach has an advantage over the flowstress approach. The example has to do with elastic precursor decay in planar impact, and with the phenomenon of anomalous thermal strengthening, revealed recently in planar impact tests. The overstress approach has an advantage whenever plastic flow during unloading is of importance.
\end{abstract}

\section{Flowstress and overstress approaches}

Dynamic viscoplastic response of ductile materials is usually modelled by assuming a constitutive law for the flowstress (Y):

$$
\begin{aligned}
& Y=Y\left(P, T, \varepsilon^{p}, \dot{\varepsilon}\right) \\
& Y^{2}=\frac{3}{2} s_{i j} s_{i j}
\end{aligned}
$$

where $\mathrm{P}=$ pressure, $\mathrm{T}=$ temperature, $\varepsilon^{\mathrm{p}}=$ effective plastic strain, $\dot{\varepsilon}=$ effective strain rate, $s_{i j}=$ stress deviator. The initial value of $\mathrm{Y}$ denoted by $\mathrm{Y}_{0}$ is called yield stress. The second equation of (1) is a closed surface in deviatoric stress space, known as the flow surface. With the flowstress approach the current stress point is always inside or on the flow surface.

Most (if not all) hydrocodes use the flowstress approach. For any computational cell, and for each time cycle, the new stress deviator is determined by a two-step algorithm introduced by Wilkins [1], and known as radial return. Step 1 calculates an intermediate stress deviator $\mathrm{s}_{\mathrm{ij}}^{*}$ and flowstress $\mathrm{Y}^{*}$, assuming that the current deformation rate is fully elastic. For $\mathrm{Y}^{*} \leq \mathrm{Y}$, the deformation is indeed elastic, and $\mathrm{s}_{\mathrm{ij}}=\mathrm{s}_{\mathrm{ij}}^{*}$. For $\mathrm{Y}^{*}>\mathrm{Y}$, the new stress deviator is on the intersection of the flow surface $\mathrm{Y}$ and the ray from $\mathrm{s}_{\mathrm{ij}}^{*}$ to the origin:

$$
\beta=Y / Y^{*} ; s_{i j}=\beta s_{i j}^{*} .
$$

To evaluate $\mathrm{Y}$ we need the new values of $\varepsilon^{p}$ and $\dot{\varepsilon}$. These are not available at this stage, and old values are used instead. This often introduces noise into the solution. It can

\footnotetext{
${ }^{a}$ Corresponding author: ypartom@netvision.net.il
}

be shown that for an unchanging flow surface, radial return is equivalent to the flow rule:

$$
d_{i j}^{p}=\lambda s_{i j}
$$

where $\mathrm{d}_{i j}^{p}$ is the plastic deformation rate, and $\lambda$ is a positive scalar. But when the flowstress changes by Eq. (1), radial return is not equivalent to Eq. (3). Equation (1) expresses isotropic hardening/softening. But it can be generalized to express also kinematic hardening and/or shape change. This has no effect on the radial return algorithm, except that in some extreme cases the ray to the origin may not cross the flow surface. Once the new $\mathrm{s}_{i j}$ are obtained, plastic deformation rate follows by:

$$
\begin{aligned}
& \delta_{i j}^{p e}=d_{i j}^{p}=\delta_{i j}-\delta_{i j}^{e} \\
& \delta_{i j}^{e}=s_{i j} / 2 G(P, T) \\
& \left(\dot{\varepsilon}_{e f f}^{p}\right)^{2}=\frac{2}{3} d_{i j}^{p} d_{i j}^{p}
\end{aligned}
$$

where $\mathrm{G}$ is the shear modulus, and $\delta_{i j}$ is the deformation rate deviator. Finally, the stress deviator is corrected for rigid body rotation by:

$$
\dot{s}_{i j}=s_{i \alpha} \omega_{\alpha j}+s_{j \alpha} \omega_{\alpha i}
$$

where $\omega_{i j}$ is the spin tensor.

We see that the flowstress approach is simple and easy to apply, and is therefore used in most if not all hydrocodes. Accordingly, most viscoplastic constitutive models have the form of Eq. (1). Also, because upon loading (deviatoric stress increase) from the flow surface, the current state becomes the new flow surface, unloading is always elastic by the flowstress approach. In

This is an Open Access article distributed under the terms of the Creative Commons Attribution License 4.0, which permits unrestricted use, distribution, and reproduction in any medium, provided the original work is properly cited. 
this respect the overstress approach is quite different from the flowstress approach.

The overstress approach is motivated by Orowan's equation, and it distinguishes the quasistatic yield stress (that sets stationary dislocations into motion) from the current stress (that maintains the motion of already moving dislocations). The quasistatic yield stress is:

$$
Y_{q s}=Y_{q s}(P, T)
$$

and the effective plastic deformation rate is given by:

$$
\begin{aligned}
& d_{e f f}^{p}=d_{e f f}^{p}\left(\sigma_{e q}-Y_{q s}\right) \\
& \sigma_{e q}=\frac{3}{2} s_{i j} s_{i j}
\end{aligned}
$$

where $\sigma_{e q-} \mathrm{Y}_{q s}$ is the overstress. The plastic deformation rate components are then given by Eq. (3) where $\lambda$ is:

$$
\lambda=\frac{3}{2} d_{e f f}^{p} / \sigma_{e q} .
$$

The rates of the deviatoric stress components are then:

$$
\begin{aligned}
& \delta_{i j}^{e}=\delta_{i j}-d_{i j}^{p} \\
& \dot{s}_{i j}=2 G(P, T) \delta_{i j}^{e}+s_{i \alpha} \omega_{\alpha j}+s_{j \alpha} \omega_{\alpha i}
\end{aligned}
$$

where the first right hand side term in the second equation of (9) is an approximation good for low deviatoric elastic strains.

We see that the overstress approach is expressed in terms of rate equations. These need to be integrated for each computational cell and for each time step separately, which can be done with a standard ODE solver like Runge-Kutta. Many times a single integration step over a computational time step is sufficient.

The deviatoric rate equations are coupled to the equation of state (EOS) both ways. First, the deviatoric part needs $\mathrm{P}$ and $\mathrm{T}$, determined from the EOS. Second, plastic work is added to the volumetric internal energy, thereby influencing $\mathrm{P}, \mathrm{T}$ and $\mathrm{V}$ (specific volume). The rate equation for $\mathrm{P}$ is:

$$
\begin{aligned}
& E=E(P, V) ; \quad \dot{E}=-P \dot{V}+\dot{W}^{p} \\
& \dot{W}^{p}=V s_{i j} d_{i j}^{p} \\
& \therefore \dot{P}=-\frac{P+\partial E / \partial V}{\partial E / \partial P} \dot{V}+\frac{\dot{W}^{p}}{\partial E / \partial P}
\end{aligned}
$$

where $\dot{V}$ during a time step is known from mass conservation, and $\mathrm{T}$ can be determined from the temperature EOS T(P,V).

Equation (7), which we call the flow function, is the main equation of the overstress approach. Its meaning is:

- In a dynamic situation the stress point is allowed to move out of the quasistatic yield surface, hence the term overstress.

- When this happens, the stress point tends to fall back (or relax) onto the quasistatic yield surface at a rate that increases with the amount of overstress.

- The rate of relaxation determines the strain rate sensitivity of the deformation.

- During plastic flow the stress point is mostly outside the quasistatic yield surface.

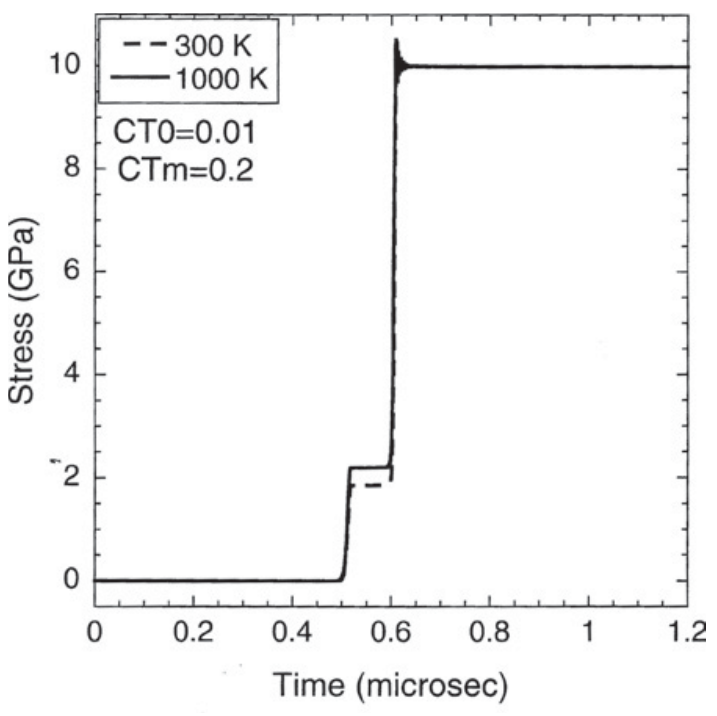

Figure 1. Longitudinal stress history at $1 \mathrm{~mm}$ into the target, calculated with the flowstress approach and a temperature dependent strain rate coefficient.

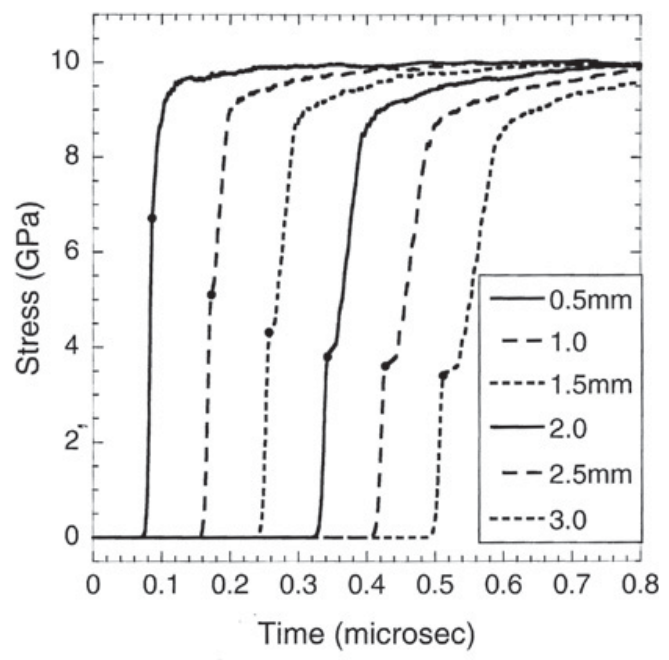

Figure 2. Longitudinal stress histories, every $0.5 \mathrm{~mm}$ into the target, calculated with the overstress approach.

- Also, plastic flow may continue upon unloading, and there is no noise from abrupt changes between elastic unloading and plastic reloading, as often happens with the flowstress approach.

We use for the flow function the simplest forms possible:

$$
\begin{aligned}
& d_{e f f}^{p}=A\left(\sigma_{e q}-Y_{q s}\right) \\
& d_{e f f}^{p}=A\left(\sigma_{e q}-Y_{q s}\right)^{\alpha} .
\end{aligned}
$$

The flow function, which reflects dislocation motion through the crystal lattice, determines both strain and strain rate hardening/softening. For a given A, when the total deformation rate is higher, there is more elastic deformation rate, and the stress increases (strain rate hardening). Also, for a given total deformation rate, when A decreases (with $\varepsilon_{\text {eff }}^{p}$ ), elastic strain rate increases (strain hardening). 


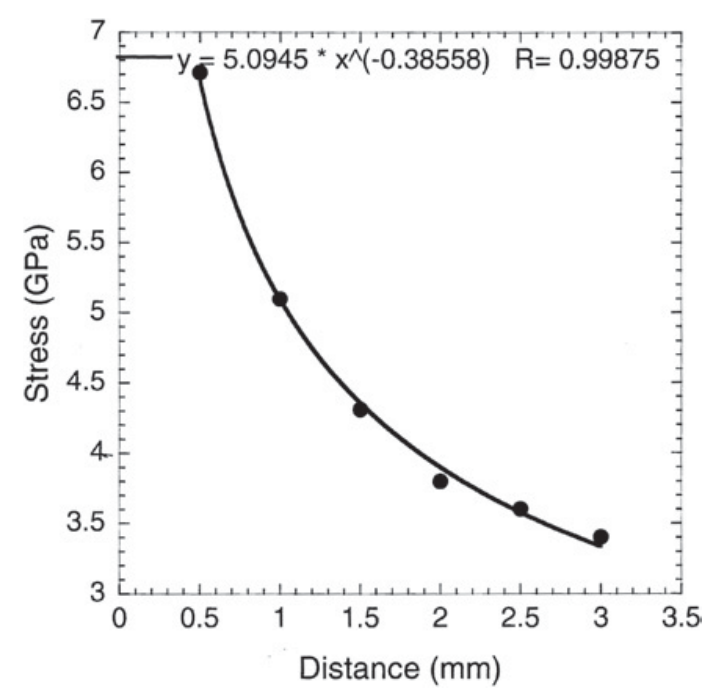

Figure 3. Precursor decay as function of distance from the calculation of Fig. 2.

In what follows we give an example for which use of the overstress approach has an advantage over the flowstress approach. The example is about elastic precursor wave decay in planar impact and the phenomenon of anomalous thermal strengthening.

\section{Anomalous thermal strengthening}

Planar impact tests on thin metal targets $(1 \mathrm{~mm}$ or less), and at different temperatures have been recently reported in [2]. In these tests the authors focused on the elastic precursor wave amplitudes, and observed that for higher temperatures the rate of elastic precursor decay is slower. Their own interpretation was that dynamic strength increases with temperature, and accordingly they referred to the phenomenon as "Anomalous Thermal Strengthening" (ATS).

Planar impact tests have been used for over 70 years to study the dynamic response of materials to high pressure shocks. In such tests a thin plate disc (the projectile) is accelerated against a thicker plate disc (the target). A shock, or a structured shock, then propagates through the target. By monitoring the velocity history of the back surface of the target on the axis, it is possible to acquire information on the pressure (EOS) and on the shear stress (strength) response of the target material. The response of viscoplastic materials to planar impact has been described and understood since the 1960s [3,4].

Dynamic response to impact (planar or non-planar) is usually simulated with a hydrocode (or wavecode), and as stated above, hydrocodes usually use the flowstress approach to calculate the shear response. As a result, hydrocodes are not able to reproduce the phenomenon of elastic precursor decay in planar impact. We show this by running a planar impact problem using the flowstress approach. At the same time we also show that ATS can be reproduced with the flowstress approach by making the strain rate term of Eq. (1) depend explicitly on temperature

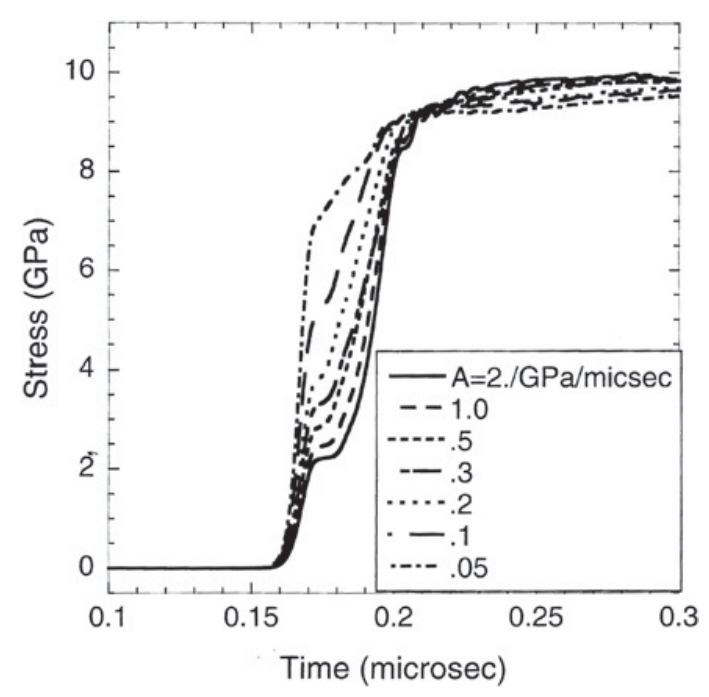

Figure 4. Longitudinal stress histories at $0.5 \mathrm{~mm}$ into the target for different values of the coefficient $\mathrm{A}$.

(in addition to the usual temperature dependence factor):

$$
\begin{aligned}
& Y=Y_{q s} Y_{P}(P) Y_{T}(T) Y_{\varepsilon}\left(\varepsilon_{e f f}^{p}\right)\left[1+C_{T}(T) \ell n \dot{\varepsilon}\right] \\
& C_{T}(T)=C_{T m}+\left(C_{T 0}-C_{T m}\right)\left(T_{m}-T / T_{m}-T_{0}\right)
\end{aligned}
$$

where the index $\mathrm{m}$ signifies melting. In Fig. 1 we show stress histories at $1 \mathrm{~mm}$ into the target from two such runs. The target is stainless steel with 100 cells per mm. The entering shock is $10 \mathrm{GPa}$. We use Yqs $=0.9 \mathrm{GPa}$, $\mathrm{G}=77 \mathrm{GPa}, \mathrm{C}_{T 0}=0.01$ and $\mathrm{C}_{T m}=0.2$. The two runs are with $\mathrm{T}=300 \mathrm{~K}$ and $1000 \mathrm{~K}$, respectively.

We see from Fig. 1 that the precursor level from the run with $1000 \mathrm{~K}$ is indeed higher. We don't show it here, but stress history curves at longer distances into the target look the same, except that the precursor wave duration is longer, and precursor decay with distance is not reproduced.

We adapted our hydrocode to use the overstress approach for the same planar impact problem. We use Eq. (11) with $\alpha=2.4$ and different values of $\mathrm{A}$. In Fig. 2 we show stress histories every $0.5 \mathrm{~mm}$ into the target and with $\mathrm{A}=0.1 / \mathrm{GPa} / \mu \mathrm{s}$. Elastic precursor decay is clearly seen.

In Fig. 3 we show the precursor decay curve as function of distance from the run of Fig. 2. In Fig. 4 we show stress history curves at $0.5 \mathrm{~mm}$ into the target for different values of the coefficient $\mathrm{A}$. For $\mathrm{A}=\mathrm{A}(\mathrm{T})$ this would be equivalent to curves of different temperatures. We see that for lower values of A (higher temperatures) precursor decay is slower, and precursor stress at a given distance is higher.

\section{Summary}

The flowstress and overstress approaches to dynamic viscoplasticity have an entirely different structure and logic, but produce equivalent results in many cases, as in monotonic loading. Flowstress is the standard approach in hydrocodes, and it is always used in conjunction with the radial return algorithm. By the flowstress approach, the flow surface in stress space is dynamic. It follows the equivalent stress upon loading, and unloading is 
always elastic. The overstress approach, on the other hand, recognizes only a quasistatic flow surface, which the stress point may move out of upon loading, and hence the term overstress. When it does, the stress point tends to fall back onto the quasistatic flow surface at a rate that increases with overstress. By the overstress approach the rate of plastic flow may be nonzero upon unloading.

The flowstress and overstress approaches may predict entirely different results whenever plastic flow during unloading is of importance. One such situation is elastic precursor wave decay in planar impact. In chapter 2 we demonstrate this by simulating a planar impact example with both approaches. We show that by using the overstress approach we can explain the anomalous thermal strengthening phenomenon recently obtained in planar impact tests of thin metal discs heated to high temperatures.

\section{References}

[1] M. Wilkins, Calculation of Elastic-Plastic Flow, LLNL Report UCRL-7322 Rev. (1969)

[2] G. I. Kanel, in Shock Compression of Condensed Matter, 937 (2011)

[3] W. Herrmann, D. L. Hicks, E. G. Young, Sock Waves and the Mechanical Properties of Solids, Syracuse Univ. Press, 23 (1971)

[4] Y. Partom, J. Appl. Phys., 59, 2716 (1986) 